\title{
CORONARY ARTERY DISEASE RISK FACTORS IN WOMEN- A STUDY ON SERUM LIPIDS AND LIPOPROTEINS
}

Genga Sadasivan Nair¹, Jessy Sumangala Janardhanan², Sreekumari Sivarama Pillai ${ }^{3}$

1 Professor, Department of Biochemistry, Government Medical College, Kollam, Kerala.

${ }^{2}$ Associate Professor, Department of Biochemistry, Government Medical College, Thiruvananthapuram, Kerala.

3 Professor and HOD, Department of Biochemistry, Sree Gokulam Medical College and Research Foundation, Thiruvananthapuram, Kerala.

\section{ABSTRACT}

\section{BACKGROUND}

Cardiovascular diseases are now the most common cause of death worldwide. Most of the risk factors for coronary artery disease were similar for both men and women, but some are unique to women. The present study was carried out to identify the presence of risk factors of CAD in a group of working women.

\section{MATERIALS AND METHODS}

A descriptive study was carried out in which distribution of serum lipids and other risk factors of CAD in 200 females of different age groups ranging from 20 to 60 years were assessed. The statistical analysis was performed using one-way ANOVA test and correlation study.

\section{RESULTS}

The mean values for lipids are total cholesterol $-184.88 \pm 30.8$, triglycerides $-100.57 \pm 38.42$, LDL cholesterol $-114.79 \pm 32.64$, HDL cholesterol - $49.95 \pm 10.5$ and total cholesterol/HDL ratio - 3.88. Comparison of the parameters in different age group shows the risk category is more in group 4, which include women in age group 46-60 yrs.

\section{CONCLUSION}

The analysis reveals that the level of total cholesterol, LDL cholesterol and triglycerides are higher in older age groups while HDL cholesterol levels are decreasing as age advances and that the women in postmenopausal age group has a more atherogenic lipid profile than premenopausal women. Those with other risk factors such as hypertension, diabetes mellitus and obesity have more atherogenic lipid profile.

\section{KEYWORDS}

Coronary Artery Disease, Lipid, Lipoprotein.

HOW TO CITE THIS ARTICLE: Nair GS, Janardhanan JS, Pillai SS. Coronary artery disease risk factors in women- A study on serum lipids and lipoproteins. J. Evolution Med. Dent. Sci. 2017;6(56):4172-4176, DOI: 10.14260/Jemds/2017/904.

\begin{abstract}
BACKGROUND
Cardiovascular disease is a major health problem in many countries. It is the leading cause of mortality in developed countries. Mortality from Coronary Artery Disease (CAD) begins later in women than in men occurring more often in postmenopausal women predominantly in their sixties and older.(1) Studies have shown a high prevalence of CAD in both urban and rural population of India. This increase in CAD rates is primarily due to the change in lifestyle, mostly the dietary changes.
\end{abstract}

\section{According to Framingham Heart Study, the risk factors for Coronary Artery Disease include-}

\section{Positive Family History}

Family history of CAD is a significant and independent risk factor for CAD.

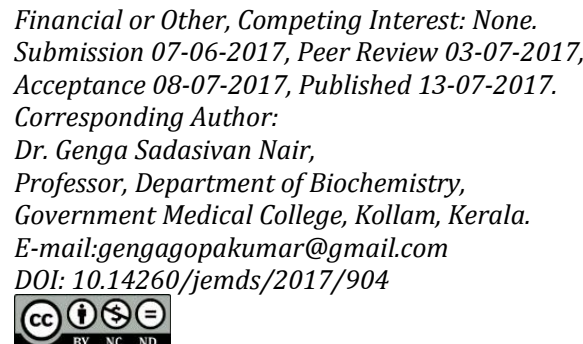

\begin{abstract}
Lipid Abnormalities
Among epidemiological and diet studies of cholesterol and cardiovascular risk in women, majority of data support elevated cholesterol as a risk factor for CAD in women. A strong correlation between HDL cholesterol and CAD is found in women. High HDL-C level in women is a strong predictor of CAD risk. ${ }^{(2,3)}$ Recent data suggests that triglyceride rich lipoproteins may play a role in atherogenesis. Hypertriglyceridaemia stimulates the formation of small dense LDL cholesterol and lowers HDL cholesterol, both of which amplify CAD risk.(4)
\end{abstract}

\section{Diabetes Mellitus}

Diabetes mellitus is a stronger risk factor for CAD in women than men. Compared to nondiabetic women, diabetic women are more likely to have abnormal lipoprotein levels with higher triglycerides and low HDL cholesterol.(5,6)

\section{Hypertension}

There is a strong association between blood pressure and CAD in both women and men. Hypertension does not initiate atherosclerotic process, but accelerates it in the presence of a threshold level of LDL cholesterol.(2)

\section{Hypoestrogenaemia}

It is a well-established fact that premenopausal women are protected against atherogenesis compared to men. Oestrogen 
acts as a protective agent against CAD. There is strong evidence that postmenopausal oestrogen deficiency increases age-specific cardiovascular risk. Epidemiological data shows that the incidence is more in postmenopausal women and young women with oestrogen deficiency either surgically induced or premature menopause. Women in this group who have undergone hormone replacement therapy have lower rates of CAD. Various studies suggest a complex relationship between CAD risk, oestrogen and menopause and serum cholesterol level in women. Oestrogen raises HDL cholesterol levels, lowers LDL cholesterol and prevents oxidative modification of LDL. Other effects of oestrogen include antioxidant property, restoration of normal endothelium, improved fibrinolysis, improved insulin sensitivity and lower Lp(a) level. $(5,6,7)$

1. Obesity worsens CAD risk factors including hypertension, diabetes and hypercholesterolaemia. In nurse's health study, women in higher category of overweight (BMI $>29$ ) experienced more than threefold risk of CAD than in those with $\mathrm{BMI}<21$. ${ }^{(8)}$

2. Physical inactivity confers a two-fold increase in risk for CAD. Exercise reduces triglyceride levels, weight and blood pressure, improves carbohydrate metabolism and enhances fibrinolytic activity. $(2,7)$

3. Stress is another risk factor for CAD, which affects the process of atherosclerosis. Chronic stress cause increased secretion of corticosteroids, LDL cholesterol and decreased HDL cholesterol.(9,10)

Recent studies show that CAD is a significant problem in India. Action must be taken to prevent CAD in both women and men before the signs and symptoms appear. It is essential to identify the prevalence of risk factors in our population to plan preventive strategies. In this study, the distribution of serum lipids and other risk factors of CAD in a group of working women were assessed.

\section{Objectives}

To study the pattern of lipid profile in women of different age group and to compare the risk factors of CAD in women of different age group.

\section{MATERIALS AND METHODS}

Descriptive study was carried out in 200 adult working women. The distribution of total cholesterol, LDL cholesterol, HDL cholesterol and triglycerides assessed. Other risk factors of CAD such as hypertension, diabetes mellitus and obesity were also analysed. The study group included students and staff of Medical College, Trivandrum. The subjects were divided into four groups based on age.

1. $15-25$ years - $(50)$.

2. 26-35 years - $(50)$.

3. $36-45$ years $-(50)$.

4. 46-60 years $-(50)$.
After getting informed consent, detailed history of each subject was taken and blood samples were collected under aseptic precautions after overnight fast of 10-12 hrs.

\section{Following Parameters were analysed in Each Sample-}

- Total cholesterol.

- Triglycerides.

- HDL cholesterol.

- LDL cholesterol.

- Fasting blood glucose.

- Body mass index.

\section{Methods}

Serum total cholesterol was estimated by enzymatic (CHODPAP) method(11,12,13) and triglycerides by GPO-PAP method.(11,14,15) HDL cholesterol was estimated after precipitation of chylomicrons, LDL and VLDL from the serum by phosphotungstate in the presence of divalent cations such as magnesium. HDL remains unaffected in the supernatant and is estimated using the protocol of cholesterol estimation.(11,16) LDL cholesterol was estimated using Friedewald's formula.(11,17)

$\mathrm{LDL}$ cholesterol $=$ total cholesterol $-(\mathrm{HDL}+$ triglyceride/5).

Fasting blood sugar(18) was assayed by the glucose oxidase method. All estimations were done in STAT FAX 2000 semi auto analyser manufactured by awareness technology. Height and weight of the subjects were measured. BMI was calculated using the formula BMI = weight in $\mathrm{kg} /$ height in $\mathrm{m}^{2}$.

\section{Statistical Analysis}

The statistical analysis was performed using one-way ANOVAs test and correlation study. Mean and standard deviation for each parameter were calculated. The distribution of total cholesterol, triglycerides, LDL cholesterol and HDL cholesterol in 200 females in different age groups ranging from 20 to 60 years was assessed and the mean values of these parameters in different age groups were compared. The measure of other risk factors of CAD such as hypertension, diabetes mellitus and obesity were also analysed.

\section{RESULTS}

Analysis of total cholesterol, LDL cholesterol and triglycerides shows higher values in older age group while HDL cholesterol is decreasing as age advances (Table 2).

\begin{tabular}{|c|c|c|c|c|c|c|}
\hline & TC & TGS & HDL & LDL & TC/HDL & LDL/HDL \\
\hline Mean & 184.88 & 100.57 & 49.95 & 114.79 & 3.88 & 2.43 \\
\hline $\begin{array}{c}\text { Std. } \\
\text { deviation }\end{array}$ & 30.81 & 38.42 & 10.5 & 32.64 & 1.27 & 1.12 \\
\hline \multicolumn{7}{|c|}{ Table 1. Mean Values of Lipids in the Total Population Studied } \\
\hline
\end{tabular}

\begin{tabular}{|c|c|c|c|c|c|c|c|c|c|c|c|c|}
\hline \multirow[t]{2}{*}{ Age } & \multicolumn{2}{|c|}{ Total Cholesterol (mg/dL) } & \multicolumn{2}{|c|}{ Triglycerides (mg/dL) } & \multicolumn{2}{|c|}{ HDL (mg/dL) } & \multicolumn{2}{|c|}{ LDL (mg/dL) } & \multicolumn{2}{|c|}{ TC/HDL } & \multicolumn{2}{|c|}{ LDL/HDL } \\
\hline & Mean & SD & Mean & SD & Mean & SD & Mean & SD & Mean & SD & Mean & SD \\
\hline Group 1 & 177.4 & 23.4 & 85.96 & 25.2 & 57.6 & 7.24 & 98.5 & 23.43 & 3.1 & 0.63 & 1.76 & 0.6 \\
\hline Group 2 & 169.04 & 22.09 & 90.6 & 30.52 & 51.6 & 8.67 & 102.06 & 22.1 & 3.31 & 0.742 & 1.93 & 0.63 \\
\hline Group 3 & 192.3 & 25.02 & 105.23 & 29.4 & 46.4 & 10.42 & 126.35 & 29.24 & 4.3 & 1.35 & 2.88 & 1.22 \\
\hline Group 4 & 200.9 & 39.7 & 120.84 & 52.9 & 43.91 & 9.89 & 132.59 & 39.4 & 4.74 & 1.35 & 3.14 & 1.2 \\
\hline
\end{tabular}




\begin{tabular}{|c|c|c|}
\hline Parameter & F & Significance \\
\hline TC & 12.714 & $<0.01$ \\
\hline TG & 9.364 & $<0.01$ \\
\hline HDL & 22.117 & $<0.01$ \\
\hline LDL & 16.912 & $<0.01$ \\
\hline TC/HDL & 27.139 & $<0.01$ \\
\hline LDL/HDL & 24.920 & $<0.01$ \\
\hline BMI & 21.091 & $<0.01$ \\
\hline \multicolumn{2}{|c|}{ Table 3. One-Way Analysis ANOVA Test } \\
\hline
\end{tabular}

\begin{tabular}{|c|c|c|}
\hline & Correlation & Significance \\
\hline Age group and TC & 0.340 & $<0.01$ \\
\hline Age group and TG & 0.347 & $<0.01$ \\
\hline Age group and HDL & -0.496 & $<0.01$ \\
\hline Age group and LDL & 0.434 & $<0.01$ \\
\hline Age group and TC/HDL & 0.525 & $<0.01$ \\
\hline Age group and LDL/HDL & 0.506 & $<0.01$ \\
\hline Age group and BMI & 0.490 & $<0.01$ \\
\hline Table 4. Correlation of Lipid Parameters with Age \\
\hline
\end{tabular}

There is an inverse correlation between age group and HDL cholesterol.

\begin{tabular}{|c|c|c|c|c|c|c|c|}
\hline Group & TC & LDL & HDL & TGS & TC/HDL & LDL/HDL & BMI \\
\hline Group 1 and 2 significance & 0.080 & 0.434 & 0.001 & 0.217 & 0.226 & 0.125 & 0.001 \\
\hline Group 1 and 3 significance & 0.002 & 0.000 & 0.000 & 0.001 & 0.000 & 0.000 & 0.000 \\
\hline Group1 and 4 significance & 0.000 & 0.000 & 0.000 & 0.000 & 0.000 & 0.000 & 0.000 \\
\hline Group 2 and 3 significance & 0.000 & 0.000 & 0.004 & 0.017 & 0.000 & 0.000 & 0.025 \\
\hline Group 2 and 4 significance & 0.000 & 0.000 & 0.000 & 0.003 & 0.000 & 0.000 & 0.000 \\
\hline Group 3 and 4 significance & 0.531 & 0.708 & 0.471 & 0.214 & 0.141 & 0.325 & 0.043 \\
\hline \multicolumn{7}{|l}{ Table 5. Comparison of Test Parameters in Different Age Groups } \\
\hline
\end{tabular}

Marked significance between the parameters of group 1 and 4 and group 1 and 3 .

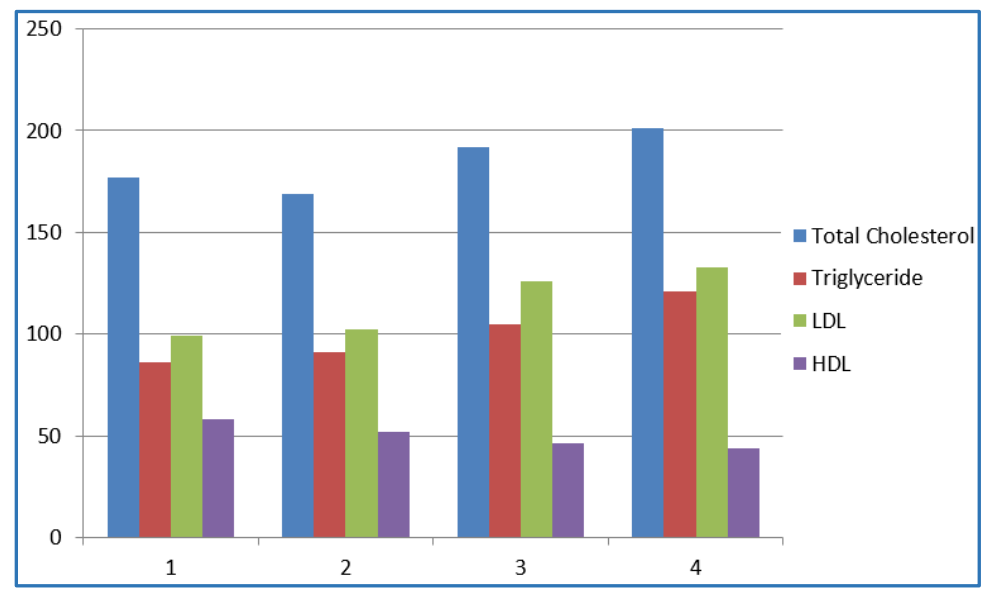

The study subjects were classified and categorised as below.

\begin{tabular}{|c|c|c|c|c|c|c|}
\hline Risk Category & Total Cholesterol & Triglyceride & LDL Cholesterol & HDL Cholesterol & TC/HDL Ratio & BMI \\
\hline Desirable & $<200$ & $<200$ & $<130$ & $>60$ & $<3.5$ & $<25$ \\
\hline Borderline & $200-239$ & $200-400$ & $130-160$ & $35-60$ & $3.5-5$ & $25-30$ \\
\hline High & $>240$ & $>400$ & $>160$ & $<35$ & $>5$ & $>30$ \\
\hline
\end{tabular}

Distribution of risk categories in various age groups was studied and it showed that the older age group has the highest risk compared to others. Twelve subjects in group 4 were hypertensive and four subjects in group 4 were diabetic.

\section{DISCUSSION}

Although, women lag behind men in incidence of coronary heart disease by 10 years, this disease is a major cause of disability and the leading cause of death in women. The advantage women have over men varies with advancing age and is to a great extent lost on attaining menopause. Evidence suggests that women with CAD have a worse prognosis when compared with men. Prevention is critical because $40 \%$ of all coronary events in women are fatal, $67 \%$ of all sudden deaths in women occur in those without a history of coronary heart disease.(19)
Longitudinal studies such as Framingham study have identified modifiable risk factors associated with the development of CAD such as hypertension, hypercholesterolaemia, obesity, smoking and a sedentary lifestyle. A number of clinical trials have established that modification of these risk factors can reduce the likelihood that an asymptomatic individual will develop CAD and the likelihood of repeat acute events such as myocardial infarction and sudden cardiac deaths in individuals with symptomatic CAD. The prognosis of coronary atherosclerosis to clinical disease occurs over many years and hence the prevention of progression of atherosclerosis would have the biggest impact on reducing the risk of CAD. The higher rates of CAD in urban India compared to rural India despite lower rates of smoking suggest important roles for nutritional and environmental factors. There is a significant increase in BMI in urban India compared to rural India. $(20,21)$ 
Little information is available about the distribution of coronary risk factors in Kerala population especially in females. It is essential to identify the prevalence of risk factors in our population to plan the preventive strategies, because according to existing knowledge, CAD epidemics are especially preventable. We have to identify who all will develop CAD, who will have accelerated progression of the disease and what are the preventive strategies that can be adopted at the beginning of disease.

In the present study, the distribution of serum lipids and other risk factors of CAD in women were assessed. The distribution of total cholesterol, HDL-C, LDLC and triglycerides in 200 females of different age groups ranging from 20-60 yrs. The presence of other risk factors of CAD such as hypertension, diabetes mellitus, obesity and dietary habits were also analysed. Risk factors of CAD in different age group of women were compared.

Analysis of total cholesterol shows higher values in older age group. Similarly, LDLC and triglyceride levels are also higher in older age group while the HDL-C levels are decreasing as age advances. The mean values of TC, TG and LDL obtained are comparable with values from other studies. The mean values obtained for the age group of $51 \pm 10$ in the Framingham offspring study (which was conducted in US white women) are total cholesterol- $206 \pm 40$, triglycerides$111 \pm 98$, LDL cholesterol- $129 \pm 37$ and HDL cholesterol- 57 \pm 15 . The mean values in older age group of this study are TC$200.96 \pm 39.7$, TGS- $120.84 \pm 52$, LDL- $132.59 \pm 39.4$ and HDL- $43.91 \pm 9.89$.

Although, one would expect a lower lipid level in our population, the values obtained are consistent with that of US women except in HDL-C levels. The mean HDL-C levels obtained in present study is $43.91 \mathrm{mg} / \mathrm{dL}$ for older age groups (range $45-60 \mathrm{mg} / \mathrm{dL}$ ), which indicates the CAD risk is more in our population since HDL-C is considered as a strong predictor of CAD in women.

When we analysed the total cholesterol/HDL-C ratio, the mean value obtained is 3.88 , and in older age group, the ratio is 4.7 ; both are higher than the optimum range 3.5 . TC/HDL ratio rises steadily with age from 3.4 at the age of 25 years to 4.7 at age 75 . When this ratio exceeds 7.5 , women have the same CHD risk as men. Ratio above 5 are dangerous and the optimal ratio is around 3.5. Previous studies have shown that TC/HDL ratio is a reliable discriminator of the presence of CAD in women regardless of the level of total cholesterol.(22,23) The relationship of serum lipid level and other risk factors shows that those with other CAD risk factors have more atherogenic lipid pattern.

When other risk factors are analysed, presence of other risk factors such as diabetes mellitus, hypertension and obesity, etc. are found in women of postmenopausal age group. Thirteen out of 50 were found to be hypertensive and 5 were found to be diabetic, 6 were found to the obese (BMI $\geq 30$ ). In these, the serum lipid pattern is undesirable. There was only one person undergoing hormone replacement therapy and hence the effect of postmenopausal oestrogen therapy could not be studied.

When the risk factors are compared in different age group, women in postmenopausal age group showed a more atherogenic lipid profile than premenopausal women. Previous studies conducted in US white women, like Framingham study, LRC follow-up study have demonstrated that, in addition to total cholesterol, HDL cholesterol, total cholesterol, HDL ratio and triglyceride levels as strong predictors of CAD in women. The present study reveals the presence of these risk factors in the postmenopausal age groups where the mean HDL cholesterol concentration is $43.91 \mathrm{mg} / \mathrm{dL}$ (desirable $>60$ ), total cholesterol HDL ratio 4.74 (optimum 3.5), while triglyceride levels are not markedly elevated. Only four women have triglyceride levels $>200$ $\mathrm{mg} / \mathrm{dL}$.

The present study also reveals that the alteration in lipid profile starts early in young age group as shown by the analysis of group 3. This rise in lipid levels can be due to the added stress that is experienced by the women in this age group.

The high rates of CAD in urban India compared to rural India despite lower rates of smoking suggests important role for nutritional, genetic and environmental factors. The excess burden of CAD in Indians is due to a combination of genetic predisposition, environmental and lifestyle factors. Adverse lifestyle factors include those associated with affluence, urbanisation and mechanisation. Decreased physical activity and increased consumption of calories and saturated fat result in abdominal obesity, insulin resistance and atherogenic dyslipidaemia.(24,25) These acquired metabolic abnormalities appear to have a synergistic effect on development of CAD in genetically predisposed individuals. In western countries, through health education efforts and behaviour modification, it has been able to bring down the serum cholesterol levels and to reduce the incidence of CAD, while our population appears to be replacing the traditional vegetarian and fish-based diet with more processed foods and meat-based diet, which maybe one of the reasons for high serum lipids and thereby high risk of CAD.

\section{CONCLUSION}

In the present study-

1. Analysis of lipid profile shows gradual increase in total cholesterol, triglycerides and LDL cholesterol with age, whereas there is a gradual decrease in the level of HDL cholesterol as age advances.

2. Triglycerides do not show significant correlation with other factors and the levels are not much elevated as shown by the previous studies in US.

3. Comparison of risk factors of CAD reveals that the presence of risk factors is more in the older age group. The alteration in the lipid profile starts early in younger age group as shown by the analysis of group 3 (35-45 yrs.).

Since, the magnitude of the problem of heart disease in women is high and the evidence that this can be decreased by a healthy lifestyle, preventive measures must be taken before women show symptoms of CAD. However, further studies in a large population are required to find out the strategies to be evolved to enable a healthy lifestyle in women.

\section{REFERENCES}

[1] Chadha SL, Radhakrishnan S, Ramachandran K, et al. Epidemiological study of coronary heart disease in urban population of Delhi. Ind J Med Res 1990;92: 424-30.

[2] Hennelkens CH. Risk factors for coronary heart disease in women. Cardiology Clinics 1998;16(1):1-8. 
[3] Manolio TA, Pearson TA, Weager NK, et al. Cholesterol and heart disease in older persons and women. Review of an NHLBI workshop. Ann Epidemiol 1992;2(12):161-76.

[4] La Rosa JC. Triglycerides and coronary risk in women and the elderly. Arch Int Med 1997;157(9):961-8.

[5] La Rosa JC. Women dyslipoproteinaemia and estrogens. Endocrinology and metabolism. Clinics of North America 1998;27(3):627-36.

[6] Kamali P, Muller T, Lang U, et al. Cardiovascular responses of perimenopausal women to hormonal replacement therapy. Am J of Obstet \& Gynec 2000;182(1):17-22.

[7] Frolkis JP. Screening for cardiovascular disease. Concepts, conflicts and consensus. Medical Clinics of North America 1999;83(6):1339-73.

[8] Manson JE, Colditz GA, Stampfer MJ, et al. A prospective study of obesity and risk of coronary heart disease in women. New Eng J Med 1990;322(13):882-9.

[9] Nathan L, Chaudhuri G. Estrogens and atherosclerosis. Ann Rev Phar Toxicol 1997;37:477-515.

[10] Merz BCN, Kop W, Krantz DS, et al. Cardiovascular stress response and CAD. Evidence of an adverse postmenopausal effect in women. Am Heart J 1998;135(5 Pt 1):881-7.

[11] Carl AB, Edward RA. Lipids lipoproteins and apo lipoproteins. Tietz Textbook of Clinical Chemistry. $3^{\text {rd }}$ edn. Philadelphia, USA: WB Saunders Company 1999:809-61.

[12] Allain CC, Poon LS, Chan CS, et al. Estimation of serum total cholesterol. Clinical Chemistry 1974:20(4):470-5.

[13] Roeschlau P, Bernt, Gruver WA. Estimation of serum total cholesterol. Clinical chemistry 1974;12:226.

[14] Young DP. Estimation of serum triglycerides. Lectures in Clinical Chemistry 1975;21:5.
[15] Fossati PP. Serum triglycerides determined colorimetrically with an enzyme that produces hydrogen peroxide. Clinical Chemistry 1982;28: 2077-80.

[16] Burstein M, Scholnic HR, Morfin R. Rapid method for the isolation of lipoproteins from human serum by precipitation with polyanions. J Lipid Res 1970;11(6):583-95.

[17] Teresa P, Mariano C, Martinez-Bru C, et al. Calculation of LDL cholesterol by using apolipoprotein $\mathrm{B}$ for classification of nonchylomicronemic dyslipidemia. Clinical Chemistry 1997;43(5):808-15.

[18] Tietz NW. Estimation of serum glucose. Fundamentals of clinical chemistry. 2nd edn. Toronto, USA: WB Saunders 1982.

[19] Eaker ED, Chesebro JH, Sacks FM, et al. Cardiovascular disease in women. Circulation 1993;88(4):1999-2009.

[20] Enas EA. Coronary artery disease epidemic in Indians: a cause for alarm and call for action. J Ind Med Assoc 2000;98(11):694-702.

[21] Reddy KS, Yusuf S. Emerging epidemic of cardiovascular disease in developing countries. Circulation 1998;97(6):596-601.

[22] Kannel WB. Metabolic risk factors for coronary heart disease in women: perspective from the Framingham study. Am Heart J 1987;114(2):413-9.

[23] Harting GH, Moore CE, Mitchell R, et al. Relationship of menopausal status and exercise level to HDL cholesterol in women. Exp Aging Res 1984;10(1):13-8.

[24] Ernst N, Fisher M, Bowel P, et al. Changes in plasma lipids and lipoproteins after a modified fat diet. Lancet 1980;316(8186):111-3.

[25] Gupta R. Prevention of coronary heart disease among Indians. Focus on primary prevention. J Ind Med Asso 2000;98(11):703-9. 